\title{
Natural Orifice Translumenal Endoscopic Surgery Inguinal Hernia Repair: A Survival Canine Model
}

\author{
Danny A. Sherwinter, MD, Amar Gupta, MD, and Jeremy G. Eckstein, MD
}

\begin{abstract}
Introduction: With over 20 million repairs performed worldwide annually, inguinal hernias represent a significant source of disability and loss of productivity. Natural orifice translumenal endoscopic surgery (NOTES $\left.{ }^{\mathrm{TM}}\right)$, as a potentially less invasive form of surgery may reduce postoperative disability and accelerate return to work. The objective of this study was to assess the safety and short-term effectiveness of transgastric inguinal herniorrhaphy using a biologic mesh in a survival canine model.

Materials and Methods: Under general anesthesia with the animal in trendelenburg position, a gastrostomy was created. A $4 \times 6 \mathrm{~cm}$ acellular dermal implant was deployed endoscopically across the myopectineal orifice, draped over the cord structures, and secured with Bioglue. Following completion of bilateral repairs the animals were survived for 14 days. At the end of the study period, the animals were euthanized and a necropsy performed. Cultures of a random site within the peritoneal cavity and at the site of implant deployment were obtained. In addition, a visual inspection of the peritoneal cavity was performed.

Results: All animals thrived postoperatively and did not manifest signs of peritonitis or sepsis at any point. At necropsy accurate placement and adequate myopectineal coverage was confirmed in all subjects. Cultures of a random site within the peritoneal cavity and at the site of implant deployment had no growth.

Discussion: This study confirms that NOTES-inguinal herniorrhaphy using a biologic implant can be performed safely. In addition, the transgastric technique provided good short-term myopectineal coverage without infectious sequelae.
\end{abstract}

\section{Introduction}

$\mathbf{W}$ ITH OVER 20 MILLION repairs performed worldwide annually, ${ }^{1}$ inguinal hernia repair represents a significant source of disability and loss of productivity. Open, tension-free, mesh repair has long been the gold standard for inguinal herniorrhaphy $(\mathrm{IH})$. This technique entails a sizeable skin incision and dissection, leading to poor cosmetic results, postoperative pain, and delayed return to activities of daily life. Because of only mild improvements in postoperative pain, disability, and increased cost, ${ }^{2}$ laparoscopic techniques for IH have not been widely accepted and the open technique remains the most common approach. ${ }^{3}$

Natural orifice translumenal endoscopic surgery (NOTES) is an attractive option for IH. Since there are no skin incisions, NOTES IH can provide a truly scarless repair and could potentially reduce pain and disability. Progress toward developing NOTES versions of totally extraperitoneal or transabdominal preperitoneal IH procedures have been hampered because these techniques require complex tissue manipulation and peritoneal dissection, simply not practical with currently available endoscopic instrumentation and platforms.

Intraperitoneal onlay mesh (IPOM) ${ }^{4,5}$ is the most straightforward method for laparoscopic IH. It involves direct placement of a mesh over the inguinal defect without reduction of the hernia sac or dissection of the peritoneum. The simplicity of IPOM IH easily lends itself to a natural orifice approach.

The aim of this study was to evaluate the short-term results of NOTES IPOM bilateral IH (BIH) in a survival canine model.

\section{Materials and Methods}

This study was approved by our institution's animal use committee. Funding for this study was provided by Lifecell Incorporated (Branchburg, NJ) as an unrestricted research grant. Additional materials were provided for this study by

Division of Minimally Invasive Surgery, Maimonides Medical Center, Brooklyn, New York. 


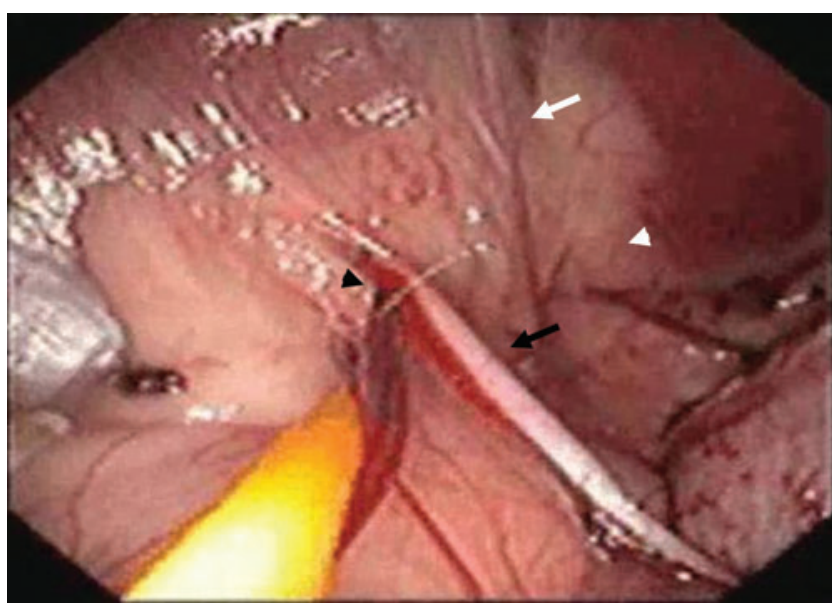

FIG. 1. Transgastric view of the left myopectineal orifice: cord structures (black arrow), internal ring (black arrowhead), external ring (white arrowhead), and inferior epigastric vessels (white arrow).

their respective manufacturers (see Disclosure Statement section).

Five male mongrel dogs weighing $20-30 \mathrm{~kg}$ were used for this study. All instruments underwent high-level disinfection with $2.4 \%$ glutaraldehyde (Cidex; Johnson \& Johnson, Somerville, NJ) before use. All subjects were maintained nil per os from midnight before surgery. A single dose of perioperative intravenous antibiotics was administered (cefazolin, 1 gram IV).

The animals were placed under general anesthesia, and gastric lavage with saline solution was performed via orogastric tube until the return was clear. As described by Onders et al., ${ }^{6}$ a Seldinger technique was used to introduce a guidewire into the gastric lumen. The wire was snared and brought out through one working channel of a dual channel gastroscope (GIF 2T100; Olympus, Tokyo, Japan). A gastrostomy was created with a sphincterotome (Hydratome RX; Boston Scientific, Natick, MA) over the guidewire. The gastrotomy was then enlarged using a $15-\mathrm{mm}$ controlled radial expansion (CRE) wireguided balloon dilator (Boston Scientific). The endoscope was then introduced into the peritoneal

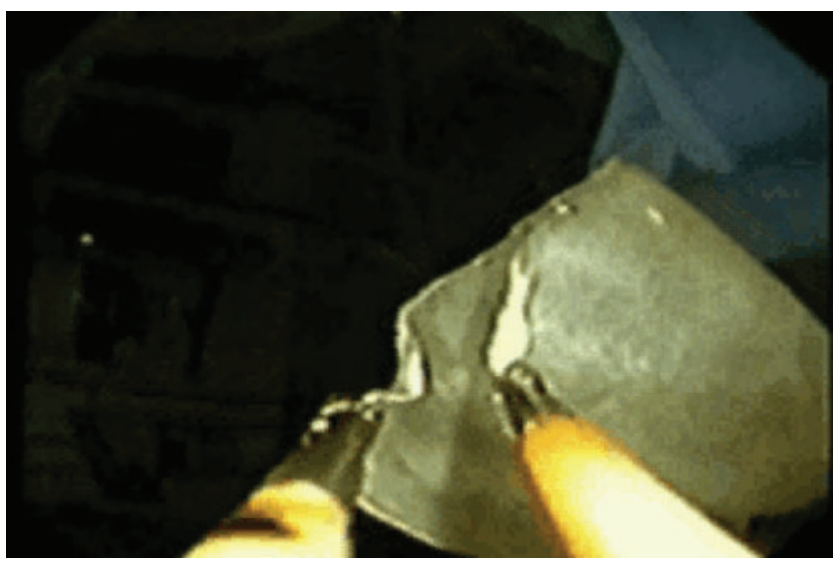

FIG. 2. Dual-channel endoscope premounted with a $4 \times 6 \mathrm{~cm}$ acellular dermal implant held by two endoscopic graspers.

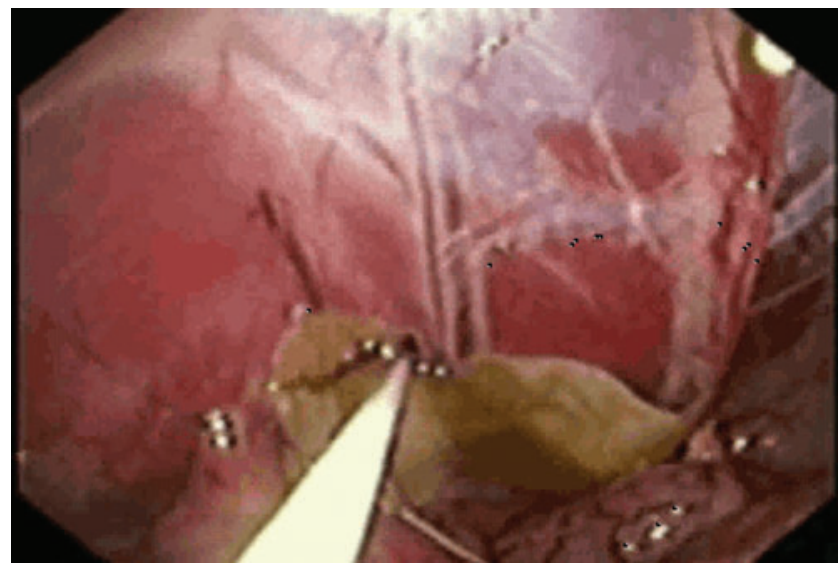

FIG. 3. The implant deployed across the myopectineal orifice and draped over the cord structures.

cavity. An overtube with an insufflation channel was used. Peritoneoscopy was performed, and bilateral deep and superficial inguinal rings were identified (Fig. 1). The scope was removed, premounted with a $4 \times 6 \mathrm{~cm}$ acellular dermal implant (AlloDerm; Lifecell) (Fig. 2), and then returned to the abdominal cavity via the overtube. The implant was then deployed across the entire myopectineal orifice and draped over the cord structures (Fig. 3). An 18-gauge needle was introduced percutaneously (Figs. 4-6), and Bioglue (CryoLife, Kennesaw, GA) was applied to secure the implant in place (Fig. 7). The gastrotomy was then closed using an endoscopic tissue approximation system (Ethicon Endosurgery, Cincinnati, $\mathrm{OH})$. Following completion of bilateral repairs the animals were survived for 14 days. At the end of the study period the animals were euthanized and a necropsy was performed. Cultures of a random site within the peritoneal cavity and at the site of implant deployment were obtained. In addition, a visual inspection of the peritoneal cavity was performed.

\section{Results}

The mean operative time for bilateral repairs was 89 minutes (range 150-45 minutes). All animals thrived postoperatively and did not manifest signs of peritonitis or sepsis at

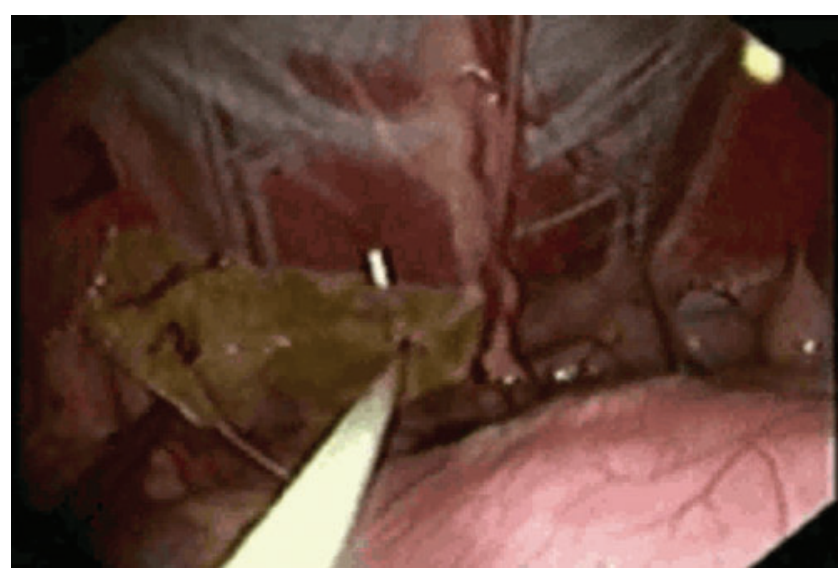

FIG. 4. Bioglue applied percutaneously to each corner of the implant sequentially. 


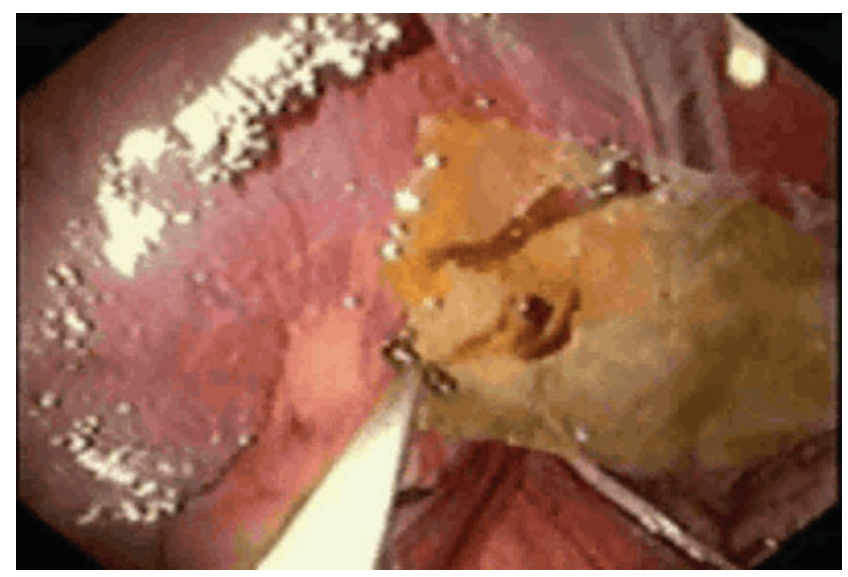

FIG. 5. Final application of the Bioglue to the lower corner of the implant completes the hernia repair.

any point. At necropsy, accurate placement and secure myopectineal coverage was confirmed in all subjects (Fig. 8). No injuries were identified at the site of gastric entry and no adhesions or abscesses were found related to the site of implantation. All cultures showed no growth.

\section{Discussion}

Primary barriers to NOTES clinical adoption include difficulty maintaining spatial orientation and the lack of an adequate multitasking platform. ${ }^{7}$ Scopes and instrumentation designed for intralumenal use are simply not up to the challenges of extralumenal surgery. Although ongoing research shows promise, ${ }^{8}$ to date even the most basic laparoscopic procedures have not made it out of the research realm.

In stark contrast to virtually all other intra-abdominal operations, IPOM BIH does not require any significant tissue manipulation. In addition, the full range of scope maneuverability, one-to-one control, and maintenance of horizon are retained in IPOM BIH since the myopectineal orifice is located directly inline with a per-orally placed scope. Because of these notable differences between IPOM BIH and other NOTES procedures, we were able to perform all of the cases in this series with a basic gastroscope and off-the-shelf endoscopic

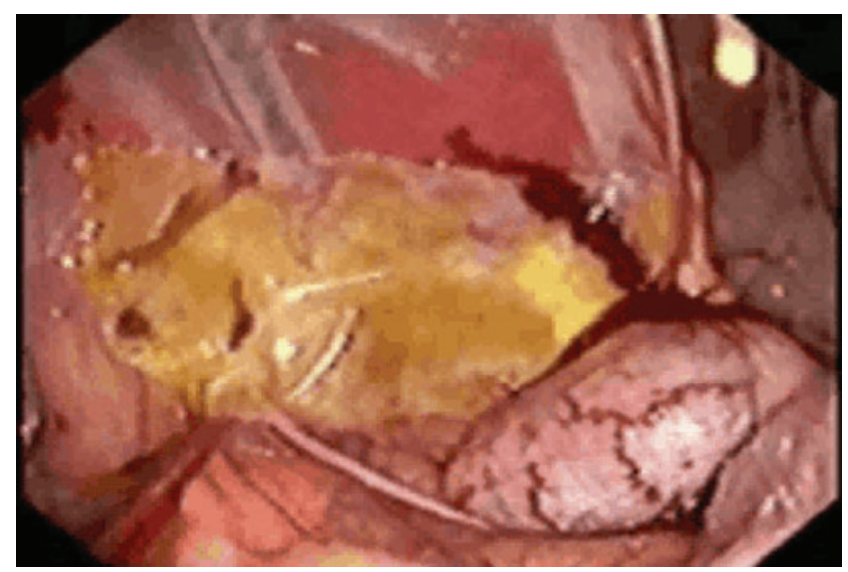

FIG. 6. Final position of the implant secured in place.

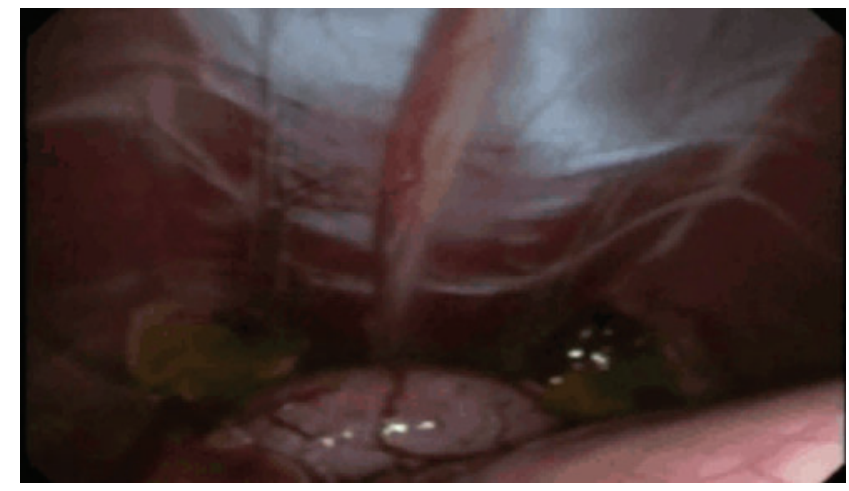

FIG. 7. Implants in position bilaterally.

instruments. In addition, operative times comparable to those seen with standard $\mathrm{BIH}$ were maintained, confirming the ready adaptability of this procedure to NOTES.

One potential shortcoming of transgastric IPOM IH is that it entails trans-oral mesh placement, which carries with it the risk of mesh infection. ${ }^{9}$ In this survival canine study we chose a biologic implant to address this issue. We confirmed neither gross nor microscopic infection at the gastrostomy or implantation site.

The use of acellular dermis (AD) for the repair of inguinal hernias in a contaminated field has been reported with excellent short-term results. ${ }^{10}$ AD retains its native extracellular matrix that promotes remodeling into vascularized tissue rather than instigating a foreign body reaction with encapsulation. Revascularization is likely the source of AD's ability to resist infection. ${ }^{11}$ Adhesion formation has also been shown to be reduced even when AD is placed in direct contact with bowel. ${ }^{12}$

This study has a number of limitations. Concerns raised by initial reports of laparoscopic IPOM include intraperitoneal adhesions and nerve and bladder injury caused by sharp intraperitoneal fixation. ${ }^{13}$ Those early studies led to a virtual moratorium on the clinical use of the IPOM technique. Recent series using biologic glues and advanced composite meshes have not replicated these findings, generating interest once

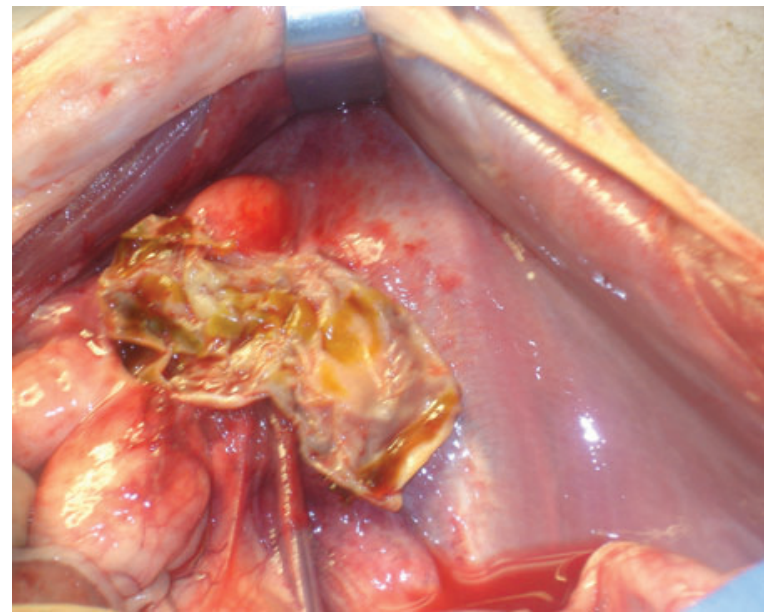

FIG. 8. At necropsy, accurate placement, and secure myopectineal coverage was confirmed in all subjects. 
again in laparoscopic IPOM repairs, ${ }^{14}$ but the long-term results of these novel IPOM techniques remain unknown.

Another limitation of this study is that Bioglue was used for mesh fixation. Although no adverse sequelae were noted in this series, Bioglue is not approved for intraperitoneal use and its effect on the abdominal viscera remains unknown. ${ }^{15}$ One published series using Bioglue on pancreatic anastamoses did not report significant intra-abdominal complications, ${ }^{16}$ but more work is needed to elucidate the role for this adhesive within the peritoneal cavity.

In conclusion, this study confirms that NOTES IPOM BIH using a biologic implant is feasible. In addition, the transgastric technique provided good short-term myopectineal coverage without infectious sequelae. NOTES IPOM BIH using a biologic implant is an interesting alternative to standard repairs and deserves further evaluation in longer-term survival studies.

\section{Acknowledgment}

This study was supported by an unrestricted research grant provided by the Lifecell Corporation. Additional study materials, including the Alloderm implants, Bioglue ampules, Hydratome RX sphinterotomes, CRE wireguided balloon dilators, and tissue approximation system, were provided gratis by their respective manufacturers.

\section{Disclosure Statement}

No competing financial interests exist.

\section{References}

1. Kingsnorth AN, LeBlanc KA. Hernias: Inguinal and incisional. The Lancet 2003;362:1561-1571.

2. Eklund A, Carlsson P, Rosenblad A, et al. Swedish Multicentre Trial of Inguinal Hernia Repair by Laparoscopy (SMIL) study group. Long-term cost-minimization analysis comparing laparoscopic with open (Lichtenstein) inguinal hernia repair. Br J Surg 2010;97:765-771.

3. Neumayer L, Giobbie-Hurder A, Jonasson O, et al. Veterans Affairs Cooperative Studies Program 456 Investigators. Open mesh versus laparoscopic mesh repair of inguinal hernia. N Engl J Med 2004;350:1819-1827.

4. Spaw AT, Ennis BW, Spaw LP. Laparoscopic hernia repair: The anatomic basis. J Laparosc Surg 1991;1:269-273.

5. Toy FK, Smoot RT. Laparoscopic hernioplasty. Surg Laparosc Endosc 1991;1:151-155.
6. Onders RP, McGee MF, Marks J, et al. Natural orifice transluminal endoscopic surgery (NOTES) as a diagnostic tool in the intensive care unit. Surg Endosc 2007;21:681-683.

7. ASGE/SAGES Working Group on Natural orifice Translumenal Endoscopic Surgery White Paper October. Gastrointest Endosc 2005;63:199-203.

8. Sherwinter DA. Two handed endoscopic robot for use in natural orifice translumenal endoscopic surgery (NOTES) transabdominal preperitoneal (TAPP) inguinal herniorrhaphy. Surg Endosc 2010;24(S1):161.

9. Kantsevoy SV, Dray X, Shin EJ, et al. Transgastric ventral hernia repair: A controlled study in a live porcine model (with videos). Gastrointest Endosc 2009;69:102-107.

10. Albo D, Awad S, Bellows DCF. Decellularized human cadaveric dermis provides a safe alternative for primary inguinal hernia repair in contaminated surgical fields. Am J of Surg 2006;192:e12-e17.

11. Diaz J, Guy J, Berkes MB, et al. Acellular dermal allograft for ventral hernia repair in the compromised surgical field. Am Surg 2006;72:1181-1187.

12. Butler CE, Prieto VG. Reduction of adhesions with composite AlloDerm/polypropylene mesh implants for abdominal wall reconstruction. Plast Reconstr Surg 2004;114:464-473.

13. Sarli L, Pietra N, Choua O, et al. Laparoscopic hernia repair, A prospective comparison of TAPP and IPOM techniques. Surg Laparosc Endosc 1997;7:472-476.

14. Olmi S, Scaini A, Erba L, et al. Laparoscopic repair of inguinal hernias using an intraperitoneal onlay mesh technique and a Parietex composite mesh fixed with fibrin glue (Tissucol). Personal technique and preliminary results. Surg Endosc 2007;21:1961-1964.

15. Hoffmann NE, Siddiqui SA, Agarwal S, et al. Choice of hemostatic agent influences adhesion formation in a rat cecal adhesion model. J Surg Res 2009;155:77-81.

16. Fisher WE, Chai C, Hodges SE, et al. Effect of BioGlue(R) on the incidence of pancreatic fistula following pancreas resection. J Gastrointest Surg 2008;12:882-890.

Address correspondence to:

Danny A. Sherwinter, MD

Division of Minimally Invasive Surgery

Maimonides Medical Center

948 48th St.

Brooklyn, NY 11219

E-mail: dsherwinter@maimonidesmed.org

\section{Commentary on "Natural Orifice Translumenal Endoscopic Surgery Inguinal Hernia Repair: A Survival Canine Model"}

I WRITE THIS COMMENTARY to congratulate the authors on this study, but more importantly, to highlight the level of disclosure they provide regarding the funding of this study and the relationship with the companies whose products are used for the study and specifically mentioned in the manuscript.

In this era of increased scrutiny of the relationships between physicians and industry, and the suggestion that too many of these relationships are tainting our ability to make appropriate decisions for our patients, ${ }^{1,2}$ this level of transparency will be increasingly important if we are to regain the trust of the regulators who are monitoring our behavior, and the patients who are beginning to ask these same questions.

Journal editors will be asking for more detail regarding funding and industry relationships before publishing re- 
search results, and the authors of this work have been very forthright in providing this important information. Readers can then interpret their results and conclusions in the context of knowing if and how various commercial interests were involved.

1. Rothman DJ, McDonald WJ, Berkowitz CD, Chimonas SC, DeAngelis CD, Hale RW, Nissen SE, Osborn JE, Scully JH Jr, Thomson GE, Wofsy D. Professional medical associations and their relationships with industry: A proposal for controlling conflict of interest. JAMA 2009;301:1367-1372.

2. Institute of Medicine (IOM) (U.S.). Conflict of interest in medical research education and practice. National Academy of Sciences, Washington, DC, 2009.

-C. Daniel Smith, MD Editor-in-Chief 
